\title{
Neophobia in taste-aversion conditioning: Individual differences and effects of contextual changes
}

\author{
TREVOR ARCHER and PER-OLOW SJÖDÉN \\ University of Uppsala, S-75104, Uppsala, Sweden
}

\begin{abstract}
In Study 1, data from a total of 260 rats in eight separate taste-aversion (saccharin + lithium chloride) experiments were analyzed for individual differences of (1) preconditioning water intake, and (2) absolute saccharin intake on the first (conditioning) and second (test) presentations; and (3) correlations between water and first saccharin intakes and between first and second saccharin intakes. A varying number of novel exteroceptive stimuli were introduced to separate groups at conditioning. The data show that individual differences of initial saccharin intake are not due to fluid intake differences in general; that initial intake values largely predict subsequent intake; and that the higher number of novel exteroceptive cues at conditioning, the more suppression of drinking is evident. Study 2 replicated the above results in a separate experiment, and supports the conclusion that the initial degree of neophobia does not affect the "associative" component in taste-aversion learning. Overall, the findings support the view that an "associative" (taste-nausea) component is superimposed on a neophobic avoidance of the novel taste+exteroceptive context in this paradigm.
\end{abstract}

In investigations of taste-aversion conditioning, very little interest seems to have been devoted to the analysis of individual differences. Furthermore, the few studies of this problem have produced conflicting conclusions. Elkins (1973) demonstrated marked individual differences of absolute saccharin intake as well as of saccharin preference after a single conditioning trial, whereas Garcia, Ervin, and Koelling (1967) reported individual differences of taste-aversion learning to be practically nonexistent. In a recent study (Archer \& Sjödén, 1979), we obtained data supporting the following conclusions: (1) There are wide individual differences among rats in the intake of a novel saccharin solution on the initial presentation (neophobia differences); (2) to a large extent, initial intake values predict the individual absolute amounts of saccharin consumed on a postconditioning presentation; and (3) the strong positive correlations between individual intakes on the first and second saccharin presentations raise serious doubts as to the appropriateness of absolute intake as a suitable measure of taste-aversion learning. When a taste suppression ratio (TSR) of individual saccharin intakes on the second to the first presentations was used as an alternative measure of learning, all animals evidenced a reduction of saccharin intake of

Thanks are due to G. Ohlin for animal caretaking. The present research was supported by Grants 1128/77, 963/78, and 584/79 from the Swedish Council for Research in the Humanities and Social Sciences and by grants from the University of Uppsala. Reprints may be obtained from Per-Olow Sjödén, Department of Psychology, Uppsala University, P.O. Box 227, S-751 04 Uppsala, Sweden. about 45\%-50\% (Archer \& Sjödén, 1979). This suggests that individual differences of taste-aversion learning may be minimal, whereas neophobia differences are of considerable magnitude.

In our previous study of this problem (Archer \& Sjödén, 1979), we were able to tentatively refute the suggestion that the individual differences of saccharin intake merely reflect individual differences of fluid intake in general rather than neophobia differences. This suggestion was rendered improbable by showing that no significant relationship existed between water intake on the day before the first saccharin presentation and subsequent saccharin intake. However, this conclusion was based on data from a rather limited number of animals ( 54 out of 222), and the study therefore needed replication.

We have previously demonstrated that the exteroceptive context present during taste-aversion conditioning and extinction trials exerts a decisive influence on the resulting aversion (Archer, Sjödén, \& Carter, 1979; Archer, Sjödén, Nilsson, \& Carter, 1979, in press). Thus, aversions were demonstrated only when the exteroceptive contexts of the conditioning and extinction trials were identical (Archer, Sjödén, Nilsson, \& Carter, 1979). It is well known that rats show neophobia not only to gustatory stimuli, but also to novel stimuli of several modalities (e.g., Barnett, 1963). Thus, we should expect a greater neophobic response, indicated, for example, by a lower intake of the solution, if a novel taste solution is presented in a novel exteroceptive context than if it is presented in a familiar exteroceptive context. In accordance with our previous finding (Archer \& Sjödén, 1979) 
that the degree of neophobia to a certain solution largely determines the intake of the solution on the postconditioning presentation, a novel-taste/novelcontext condition on the conditioning trial should yield a lower subsequent absolute intake than a noveltaste/familiar-context condition. This prediction is in line with the conception proposed by Mitchell and his collaborators (e.g., Mitchell, Hoch, \& Fitzsimmons, 1975; Mitchell, Parker, \& Johnson, 1976), that the associative taste-illness component is superimposed on a neophobic reaction to the novel taste + exteroceptive context in taste-aversion conditioning.

In the present study, we report additional data on: (1) the correlation between individual intakes of water (on the day before a taste-aversion conditioning trial) and saccharin (on the conditioning trial)-our hypothesis was that the individual values of saccharin intake are independent of preconditioning water intake; and (2) the correlation between intakes of saccharin on the conditioning trial and on a postconditioning presentation-on the basis of our previous findings (Archer \& Sjödén, 1979), we predicted a positive relationship between these variables. The present study also examines the prediction that an increasing number of novel exteroceptive stimuli, introduced on the conditioning trial, would yield a progressively lower absolute saccharin intake.

\section{STUDY 1}

The data reported are based on observations of: (1) water and saccharin intake on 2 consecutive days by a total of 134 rats in six separate experiments; and (2) saccharin intake by a total of 126 rats on a conditioning trial and the subsequent postconditioning saccharin presentation, in five separate experiments. In some of the experiments, a varying number of novel exteroceptive stimuli were introduced on the conditioning trial. Although the experiments were originally designed to test several different hypotheses, they share all procedural elements neces- sary for an examination of the problems raised in the present paper.

\section{Method}

Subjects

All 260 subjects were male Sprague-Dawley rats (AB Anticimex, Sollentuna, Sweden), weighing approximately $225 \mathrm{~g}$ (range 200$250 \mathrm{~g}$ ) at the beginning of the experiments. Within each experiment, all groups ( $n=6$ or 8 ) were matched for body weight. All animals received a 2-week acclimatization period to laboratory conditions. They were kept throughout on a 12-h-on/12-h-off (lights on at $0800 \mathrm{~h}$ ) lighting schedule in a room thermostatically maintained at $22^{\circ} \pm 1^{\circ} \mathrm{C}$. Ad-lib food (lab chow R3, Astra/ Ewos, Södertälje, Sweden) was available throughout the experiments.

\section{Apparatus}

The exteroceptive context in which the saccharin/water presentations were made consisted of three components which varied between the experimental conditions (see Table 1): (1) housing-either "contextual" compartments $(15 \times 35 \times 20 \mathrm{~cm})$ consisting of opaque plastic boxes with light excluded by Masonite covers, except for two small holes for the drinking bottles, or "normal" individual perspex cages $(16.5 \times 22.5 \times 13.5 \mathrm{~cm})$ with gridded metal covers with spaces for food and a water bottle; (2) drinking bottles-either "noisy" bottles (Techniplast, Italy) with metal nozzles with a 6-mm hole at the tip and containing a small metal ball, which created a significant amount of noise when the animals licked the tops of the nozzles, or "silent" bottles consisting of standard glass bottles with metal nozzles and a 2-mm opening at the tip; and (3) odor-either a distinctive mentholated odor (Vicks Vapo Rub, Richardson-Merrell, London), impregnated on the inside of the "contextual" compartments or on wooden boards outside the cages, or no particular odor. Table 1 specifies the exteroceptive context used for each experimental condition on Days 1-3. The number of novel exteroceptive stimuli introduced on the conditioning trial is given by the label for each condition (e.g., $\mathrm{A} 0=$ none, $\mathrm{Cl}=$ one).

\section{Procedure}

Water presentation (Day 1). All animals received between 4 and 6 days of training to a $30-\mathrm{min} /$ day water drinking schedule, beginning with a 60 -min exposure that was subsequently reduced to $30 \mathrm{~min}$. This training took place under conditions that were identicial to those for acclimatization ("normal" cages, "silent" bottles, no odor), except for Condition BO (Table 1), in which the animals were placed in "contextual" compartments and received water from "noisy" bottles in the presence of the odor, and Condition $\mathrm{Cl}$, in which the animals (Table 1) received water

Table 1

Exteroceptive Contexts and Drinking Fluids Employed in the Experimental Conditions of Studies 1 and 2

\begin{tabular}{cccc}
\hline Condition & \multicolumn{1}{c}{ Day 1: Water } & Days 2-3: Saccharin/Water & Fluid \\
\hline & & Study 1 & Saccharin \\
A0 & Normal, Silent, No Odor & Normal, Silent, No Odor & Saccharin \\
B0 & Contextual, Noisy, Odor & Contextual, Noisy, Odor & Saccharin \\
C1 & Normal, Noisy, No Odor & Normal, Noisy, Odor* & Saccharin \\
D2 & Normal, Silent, No Odor & Contextual, Noisy, No Odor & Saccharin \\
E3 & Normal, Silent, No Odor & Contextual, Noisy, Odor & Water \\
F3 & Normal, Silent, No Odor & Contextual, Noisy, Odor & Saccharin \\
G1 & Normal, Silent, No Odor & Contextual, Noisy, No Odor & Saccharin \\
H2 & Normal, Silent, No Odor & & \\
& & Study 2 No Odor & Saccharin \\
FAM & Normal, Silent, No Odor & Normal, Silent, No Odor & Saccharin \\
NOV & Normal, Silent, No Odor & Contextual, Noisy, No Odor & \\
\hline
\end{tabular}

Note-Normal = "normal" cage ; contextual = "contextual" compartment ;ilent = "silent" bottle; noisy = "noisy" bottle .

*The particular novel exteroceptive stimuli introduced on Day 2 are indicated by italics. 
from "noisy" bottles. The last day of water drinking is designated as Day 1, data from which will be used in the present study.

Saccharin/water presentation (Day 2). On Day 2, 30-min saccharin/water presentations were made either in the presence of the same set of exteroceptive stimuli as for Day 1 (Conditions $\mathrm{A} 0$ and $\mathrm{B} 0$ ) or in the presence of a varying number of novel contextual stimuli (Conditions C1, D2, E3, F3, G1, and H2).

The Day 2 presentations were performed $24 \mathrm{~h}$ after the Day 1 presentations. Condition A0 rats remained in the "normal" cages in an odor-free room, while Condition BO rats remained in "contextual" compartments in the presence of the odor. The animals in Conditions D2, E3, and F3 were moved to "contextual" compartments, and those in Conditions $\mathrm{C} 1, \mathrm{E} 3$, and F3 were exposed to the odor; Condition D2 rats remained in an odor-free room. Following these manipulations, the rats were offered saccharin (.2\% solution) in "noisy" bottles in Conditions B0, C1, D2, and E3 and in "silent" bottles in Condition A0. The animals in Condition F3 received water in "noisy" bottles. Thus, while the rats in Conditions $\mathrm{A} 0$ and $\mathrm{B} 0$ were given a novel saccharin solution in the presence of the same set of exteroceptive stimuli as for water drinking on Day 1, those in Conditions C1, D2, and $\mathrm{E} 3$ were given the novel solution in the presence of a novel odor (C1), a novel compartment + bottle (D2), or a novel compartment + bottle + odor (E3). Rats in Condition F3 received water in the presence of novel compartment + bottle + odor stimuli. In the remaining two conditions (G1 and H2), either compartments or compartments + bottles were novel on Day 2. Thus, rats in Condition G1 received saccharin in "silent" bottles in the "contextual" compartments in an odor-free room. Condition $\mathrm{H} 2$ animals were placed in compartments and received saccharin in "noisy" bottles in an odor-free room.

Approximately $15 \mathrm{~min}$ after the saccharin/water presentations, each rat in Conditions A0, B0, F3, G1, and $\mathrm{H2}$ was given an intraperitoneal (IP) $2.5-\mathrm{ml}, .15-\mathrm{M} \mathrm{LiCl}$ injection and was immediately replaced in its particular experimental environment, where it remained for the rest of the experiment.

Saccharin/water presentation (Day 3). Twenty-four hours after the Day 2 presentations, the rats in Conditions A0, B0, and $\mathrm{H} 2$ were offered saccharin for $30 \mathrm{~min}$ after having been handled. The second saccharin/water presentation to the F3 (water) and G1 (saccharin) conditions took place $48 \mathrm{~h}$ after the Day 2 presentation, and a 30-min period of water drinking in cages with "silent" bottles in an.odor-free room was interposed on the day between. The exteroceptive contexts were identical to those of Day 2 for all groups.

\section{Results \\ Day 1 Water-Day 2 Saccharin/Water \\ Presentations}

Table 2 (column 9) shows product moment correlations between Day 1 water intakes and Day 2 saccharin (Conditions A0-E3) or water (Condition F3) intakes. All correlations were positive but failed to reach statistical significance. The overall correlation based on intake data from all 116 animals in Conditions A0-E3 was +.055 . These data amply confirm our previous conclusion (Archer \& Sjödén, 1979) that individual differences of intake on the first saccharin presentation do not reflect individual differences of fluid intake in general.

As a measure of the neophobic response to the novel saccharin solution + exteroceptive context, we computed drinking suppression ratios (DSRs) (cf. Lyon, 1968) as the ratios of the amounts of Day 2 saccharin (A0-E3) or water (F3) to Day 1 water consumed by individual animals (see Table 2, column 10). An analysis of variance of a completely randomized design with unequal groups yielded a significant effect of Conditions $[F(5,128)=10.1, p<.01]$. Subsequent pairwise between-conditions $t$ tests (Kirk, 1968 , p. 74) indicated significant A0, B0, C1, F3 $>$ D2, E3 differences ( $t$ values from 2.4 to $4.1, \mathrm{df}=$ $128, \mathrm{p}<.001$ ). Also, Condition F3 animals showed a significantly higher DSR value than the combined values of Conditions $\mathrm{A} 0+\mathrm{B} 0+\mathrm{C} 1(\mathrm{t}=5.63, \mathrm{df}=$ $128, \mathrm{p}<.001)$. These results indicate that the presentation of the novel taste stimulus suppressed drinking significantly in the saccharin conditions (A0-E3) as compared to the water condition (F3). Furthermore, a greater suppression of drinking was evidenced by those saccharin conditions where two or three sets of exteroceptive stimuli were novel on Day 2 (D2 and E3) as compared to those where only one set of stimuli

Table 2

Mean Water (W) and Saccharin (S) Intake Values (in Grams), Correlations, and Mean Drinking and Taste Suppression Ratios in Studies 1 and 2

\begin{tabular}{|c|c|c|c|c|c|c|c|c|c|c|c|c|c|}
\hline \multirow{2}{*}{$\begin{array}{l}\text { Condi- } \\
\text { tion }\end{array}$} & \multirow[b]{2}{*}{$\mathrm{N}$} & \multicolumn{2}{|c|}{ Day 1: W Intake } & \multicolumn{3}{|c|}{ Day 2: S/W Intake } & \multicolumn{3}{|c|}{ Day 3: S/W Intake } & \multicolumn{2}{|c|}{ Day 1-Day 2} & \multicolumn{2}{|c|}{ Day 2-Day 3} \\
\hline & & Mean & SD & Fluid & Mean & SD & Fluid & Mean & SD & $\mathrm{r}$ & DSR & $\mathbf{r}$ & TSR \\
\hline \multicolumn{14}{|c|}{ Study 1} \\
\hline A0 & 18 & 17.7 & 1.8 & $\mathbf{S}$ & 16.9 & 3.7 & $\mathrm{~S}$ & 6.9 & 3.5 & +.032 & .93 & $+.603 *$ & .40 \\
\hline B0 & 24 & 15.5 & 4.8 & S & 12.9 & 3.5 & $\mathrm{~S}$ & 7.5 & 2.4 & +.387 & .87 & $+.611^{*}$ & .56 \\
\hline $\mathrm{Cl}$ & 32 & 18.3 & 2.5 & S & 15.0 & 4.9 & & & & +.045 & .83 & & \\
\hline D2 & 24 & 18.0 & 2.3 & S & 10.4 & 3.1 & & & & +.158 & .57 & & \\
\hline E3 & 18 & 17.7 & 2.2 & $\mathrm{~S}$ & 11.6 & 2.0 & & & & +.313 & .65 & & \\
\hline F3 & 18 & 17.1 & 3.3 & W & 17.4 & 1.5 & W & 13.5 & 3.0 & +.270 & 1.00 & +.395 & .78 \\
\hline G1 & 48 & & & $\mathrm{~S}$ & 12.6 & 3.6 & S & 4.9 & 2.4 & & & $+.604 *$ & .40 \\
\hline $\mathrm{H} 2$ & 18 & & & $S$ & 10.1 & 3.4 & $S$ & 4.1 & 2.4 & & & $+.870 *$ & .40 \\
\hline \multicolumn{14}{|c|}{ Study 2} \\
\hline FAM & 10 & 18.2 & 2.6 & S & 13.5 & 2.9 & $\mathrm{~S}$ & 5.6 & 1.3 & +.000 & .75 & +.195 & .42 \\
\hline NOV & 10 & 19.6 & 2.0 & $\mathrm{~S}$ & 9.8 & 2.2 & $\mathrm{~S}$ & 3.0 & 1.2 & +.051 & .50 & +.568 & .35 \\
\hline
\end{tabular}

Note-Day 2 = first presentation; Day 3 = second presentation. Mean DSRs consist of ratios between amounts drunk on Day 2 (saccharin/water) and Day 1 (water) presentations for individual animals. Mean TSRs consist of ratios between amounts drunk on Day 3 and Day 2 (saccharin/water) presentations for individual animals. Cells left blank indicate no data were available. ${ }^{*} p<.01$. 
(C1) or no contextual stimuli were novel (A0 and B0).

\section{Day 2-Day 3 Saccharin/Water Presentations}

Table 2 (column 11) shows product moment correlations between Day 2 and Day 3 saccharin (Conditions A0, B0, G1, H2) or water (F3) intakes. All correlations were positive and, with the exception of Condition F3, statistically significant. The overall correlation based on data from all 108 rats in the saccharin conditions was $+.635(p<.01)$. These findings confirm our previous conclusion (Archer \& Sjödén, 1979) that there is a strong positive correlation between the amounts consumed on the first and second saccharin presentations under these experimental conditions.

As an alternative to absolute saccharin intake as a measure of learning, we computed taste suppression ratios (TSRs) (cf. Archer \& Sjödén, 1979) as the ratios of the amounts of saccharin (Conditions A0, $\mathrm{B} 0, \mathrm{G} 1, \mathrm{H} 2)$ or water (F3) consumed by individual animals on the second and first presentations, thus correcting for individual neophobia differences. Mean TSRs are shown in Table 2, column 12. It is evident that intake decreased to a similar degree from the first to the second presentation in all conditions, except B0 and F3. An analysis of variance of a completely radomized design with unequal groups yielded a significant Conditions effect $[F(4,121)=23.0, p<$ .01]. Subsequent pairwise $t$ tests (Kirk, 1968, p. 74) indicated significant $\mathrm{F} 3>\mathrm{A} 0, \mathrm{~B} 0, \mathrm{G} 1$, and $\mathrm{H} 2$ ( $\mathrm{t}$ values from 4.3 to $5.5, \mathrm{df}=121, \mathrm{p}<.001$ ) and $\mathrm{B} 0>\mathrm{A} 0, \mathrm{G} 1$, and $\mathrm{H} 2$ ( $\mathrm{t}$ values from 3.1 to 5.3 , df $=121, \mathrm{p}<.001)$ differences. These data suggest that saccharin drinking was suppressed significantly more after a contingent taste-poison pairing (Conditions A0, B0, G1, H2) than water drinking after a water-poison pairing (Condition F3). However, the TSR-value for Condition F3 is well below 1.00 , which suggests that an aversion to the water + exteroceptive context complex was formed.

The B0 > A0, G1, H2 differences suggest variations in the "conditionability" of saccharin aversions. However, the TSR data do not form a pattern which is related to the relative degree of novelty of the taste + exteroceptive context of the conditioning trial. It should be noted that our prediction of the effects of the degree of initial neophobia was stated with regard to absolute intake, and not to TSRs. Separate analyses of variance of absolute saccharin intake for Day $2[\mathrm{~F}(3,104)=17.5, \mathrm{p}<.01]$ and Day $3[\mathrm{~F}(3,104)=9.6, \mathrm{p}<.01]$, and subsequent pairwise $t$ tests revealed higher intakes for the $\mathrm{A} 0$ and B0 conditions (no novel stimuli at the conditioning trial) as compared to Conditions $\mathrm{G} 1$ and $\mathrm{H} 2$ (novel compartments and novel compartments + bottles, respectively), except for a nonsignificant $\mathrm{B} 0>\mathrm{G} 1$ difference on Day 2 ( $t$ values from 2.74 to $6.18, \mathrm{df}=$
104, $\mathrm{p}<.01$ ) (see Table 2). Thus, although DSR data are lacking for $\mathrm{G} 1$ and $\mathrm{H} 2$, the findings suggest support for the contention that the more neophobia shown to the taste + exteroceptive context of the conditioning trial, the lower the absolute saccharin intake on a subsequent trial. However, this should not be taken to indicate that taste-aversion learning is thus affected, since TSR data do not form a pattern consistent with this prediction.

The next study consisted of an experiment designed explicitly to further investigate (1) whether the presence of a number of novel exteroceptive stimuli at conditioning would result in a lower intake of the novel taste substance; and (2) what, if any, relationship held between the initial degree of neophobia to the taste + exteroceptive context, on the one hand, and subsequent absolute saccharin intake and TSR, on the other.

\section{STUDY 2}

\section{Subjects}

\section{Method}

Twenty experimentally naive male Sprague-Dawley rats with a mean body weight of $275 \mathrm{~g}$ (range 250-300 g) were assigned to two groups $(n=10)$, matched for body weight.

They were kept under the identical environmental conditions as in the experiments of Study 1, with the exception that a 1month acclimatization period was employed. Following this, the animals received 6 days of training to a $30-\mathrm{min} /$ day water drinking schedule. During laboratory adjustment and drinking training, all animals were housed in the "normal" cages under odor-free conditions and received water in "silent" bottles.

\section{Apparatus}

A combination of "contextual" compartments and "noisy" bottles was utilized as the novel exteroceptive context at the conditioning trial for one of the groups (NOV) (see Table 1).

\section{Procedure}

On Day 2, $24 \mathrm{~h}$ after the last day of water drinking (Day 1), Group NOV (novel) animals were placed in "contextual" compartments and were moved to a separate room, while those of the FAM (familiar) group were handled and remained in the "normal" cages. All animals were then offered a $.2 \%$ saccharin solution, Group NOV from "noisy" bottles and Group FAM from "silent"' bottles. Approximately $15 \mathrm{~min}$ after the saccharin drinking period, all animals received an IP 3.0-ml, .15-M LiCl injection and were replaced in their experimental environments. On Day 3, a second saccharin presentation, identical to the first, was performed.

\section{Results \\ Day 1 Water-Day 2 Saccharin Presentations}

Mean Day 1 water intake, Day 2 saccharin intake, and product moment correlations between Day 1 water and Day 2 saccharin intakes are presented in Table 2 (columns 3, 5, and 9 , respectively). The overall correlation based on data from all 20 animals was +.205 . None of the correlations were significant, showing, again, that individual differences of initial saccharin intake are not merely reflections of fluid intake differences in general. 
DSRs for Groups NOV and FAM are given in Table 2 (column 10). A t test (Kirk, 1968, p. 74) indicated a significant FAM $>$ NOV difference $(t=$ 3.54 , df $=18, \mathrm{p}<.01$ ), which confirms the finding in Study 1 of a greater suppression of saccharin drinking in the presence of novel compartment + bottle stimuli than in the absence of any explicit contextual change.

\section{Day 2-Day 3 Saccharin Presentations}

Mean values of saccharin intake on Day 3 are presented in Table 2 (column 7). Analysis of variance of Day 2 and Day 3 data in a split-plot design resulted in a significant Groups effect $[\mathrm{F}(1,18)=18.0, \mathrm{p}<$ $.01]$. Thus, Group NOV drank significantly less saccharin than Group FAM on both days as a result of the novel exteroceptive cues of the conditioning trial.

Product moment correlations between mean Day 2 and Day 3 saccharin intakes are shown in Table 2 (column 11). The overall correlation based on data from all 20 rats was +.621 . Only the overall correlation was significant. TSRs are shown in Table 2 (column 12). These values were not significantly different $(\mathrm{t}=1.49$, df $=19, \mathrm{p}>.10)$. The significant overall correlation confirms our previous conclusion that initial saccharin intake data to a large extent predict postconditioning saccharin intake (Archer \& Sjödén, 1979; Study 1). Since TSRs did not differ, the introduction of a novel contextual environment at the conditioning trial, resulting in a greater suppression of absolute saccharin intake in Group NOV than a no-change condition in Group FAM, does not seem to influence the "associative" component in taste-aversion conditioning (cf. Mitchell, Kirschbaum, \& Perry, 1975; Mitchell, Scott, \& Mitchell, 1977). Furthermore, the absence of a significant NOV-FAM difference of TSRs suggests that the B0 > A0, G1, H2 differences, found in Study 1, may be either spurious or due to the fact that Condition B0 animals were the only ones that were placed in "contextual" compartments with "noisy" bottles and odor during the preconditioning period. Only further investigations can clarify this issue.

\section{DISCUSSION}

The data from the present studies lead to the following conclusions: (1) Individual differences of intake on the first presentation of saccharin do not reflect individual differences of fluid intake in general. Rather, the saccharin intake differences are more profitably regarded as individual differences of neophobia to the taste + exteroceptive context. (2) Individual intake values from the initial presentation of saccharin to a large extent predict intake of saccharin on the first postconditioning saccharin presen- tation. Taste suppression ratios (TSRs) are suggested as an alternative to absolute intake as a measure of the "associative" component in taste-aversion conditioning. These two conclusions confirm previous findings (Archer \& Sjödén, 1979). (3) The introduction of an increasing number of novel exteroceptive stimuli at the conditioning trial results in a progressively lower intake of the novel saccharin substance. Drinking suppression ratios (DSRs) may be employed as a measure of neophobia to the taste + exteroceptive context complex. (4) The hypothesis that a noveltaste/novel-context condition at the conditioning trial should yield a lower postconditioning absolute intake of the taste substance than would a noveltaste/familiar-context condition was supported. These data further support and extend recent findings by Braveman (1978) that preexposure to novel exteroceptive stimuli may totally disrupt subsequent neophobia to saccharin. It should be noted that no consistent relationship between DSR and TSR data was evident in any of the present studies, indicating that variations in the neophobia to a taste + context combination do not affect the "associative" component in taste-aversion learning. A similar independence of neophobia and taste-aversion learning has been reported recently (Braveman \& Jarvis, 1978).

In a previous study (Archer \& Sjödén, 1979), we suggested the TSR as a measure of the change of the "value" of the taste stimulus caused by the conditioning trial. In the present studies, we manipulated the novelty of the taste + exteroceptive context at the conditioning trial, and demonstrated predictable degrees of neophobic suppression of saccharin drinking (see DSRs in Table 2, column 10). The lack of a consistent relationship between DSRs and TSRs may be considered an indirect validation of the TSR as a measure of the "associative" component in tasteaversion learning. Also, the findings of TSR values of 1.43 and 1.66 , respectively, for placebo $(\mathrm{NaCl}$ instead of $\mathrm{LiCl}$ ) and unpaired ( $\mathrm{LiCl} 24 \mathrm{~h}$ prior to the first saccharin presentation) conditions in a previous study (Archer \& Sjödén, 1979) provides additional support for the TSR as an "associative" measure. Further investigations should be directed at how the TSR is affected by variables known to be related to "associative" learning processes, for example, the number of conditioning trials and stimulus intensity.

\section{REFERENCES}

Archer, T., \& Suödén, P. O. Positive correlation between preand postconditioning saccharin intake in taste-aversion learning. Animal Learning \& Behavior, 1979, 7, 144-148.

Archer, T., Suödén, P. O., \& Carter, N. Control of tasteaversion extinction by exteroceptive cues. Behavioral and Neural Biology, 1979, 25, 217-226.

Archer, T., Suödén, P. O., Nilsson, L.-G., \& Carter, N. Role of exteroceptive background context in taste-aversion con- 
ditioning and extinction. Animal Learning \& Behavior, 1979, 7, 17-22.

Archer, T., Suödén, P. O., Nilsson, L.-G., \& Carter, N. Exteroceptive context in taste-aversion conditioning and extinction: Odour, cage, and bottle stimuli. Quarterly Journal of Experimental Psychology, in press.

Barnett, S. A. The rat: A study in behaviour. London: Methuen, 1963.

Braveman, N. S. Preexposure to feeding-related stimuli reduces neophobia. Animal Learning \& Behavior, 1978, 6, 417-422.

Braveman, N. S., \& JARVIS, P. S. Independence of neophobia and taste-aversion learning. Animal Learning \& Behavior, 1978, 6, 406-412.

ElKins, R. L. Individual differences in bait shyness: Effects of drug dose and measurement technique. Psychological Record, 1973, 23, 349-358.

Garcia, J., Ervin, F., \& Koelling, R. Bait shyness: A test for toxicity with $N=2$. Psychonomic Science, 1967, 7, 245-246.

KIRK, R. E. Experimental design: Procedures for the behavioral sciences. Belmont, Calif: Brooks/Cole, 1968.
Lyon, D. O. Conditioned suppression: Operant variables and aversive control. Psychological Record, 1968, 18, 317-338.

Mitchell, D., Hoch, N. E., \& Fitzsimmons, M. Effects of neophobia sensitization on the rat's preference for earned food. Behavioral Biology, 1975, 13, 519-525.

Mitchell, D., Kirschbaum, E. H., \& Perry, R. L. Effects of neophobia and habituation on the poison-induced avoidance of exteroceptive stimuli in the rat. Journal of Experimental Psychology: Animal Behavior Processes, 1975, 1, 47-55.

Mitchell, D., Parker, L. F., \& Johnson, R. Absence of a generalization decrement in the poison-induced avoidance of interoceptive stimuli in the rat. Physiological Psychology, 1976, 4, 121-123.

Mitchell, D., Scott, D. W., \& Mitchell, L. K. Attenuated and enhanced neophobia in the taste-aversion "delay of reinforcement" effect. Animal Learning \& Behavior, 1977, 5, 99-102.

(Received for publication February 15, 1979; revision accepted September 19, 1979.) 